\title{
Convergent Validity of the Cognitive Performance Scale of the interRAI Acute Care and the Mini-Mental State Examination
}

\author{
Natbalie I.H. Wellens, Ph.D., S.L.P., M.Sc., Joban Flamaing, M.D., Ph.D., \\ Jos Tournoy, M.D., Ph.D., Tina Hanon, R.N., M.S.N., Pbilip Moons, Ph.D., R.N., \\ Geert Verbeke, Ph.D., Steven Boonen, M.D., Ph.D., Koen Milisen, Ph.D., R.N.
}

\begin{abstract}
Objective: The Cognitive Performance Scale (CPS) is generated from five items of the interRAI/Minimum Data Set instruments, a comprehensive geriatric assessment method. CPS was initially designed to assess cognition in residential care, where it has shown good psychometric performance. We evaluated the performance of the interRAI Acute Care in identifying cognitive impairment among patients hospitalized on acute geriatric wards. Methods: An observational study was conducted on two geriatric wards. Trained raters independently completed the interRAI Acute Care and the Mini-Mental State Examination (MMSE) in 97 inpatients ( $85 \pm 5$ years; $67 \%$ female). The level of agreement between CPS and MMSE was explored using comparisons of means, agreement coefficients, and diagnostic accuracy. Results: Cognitive impairment was present in $61 \%$ of the participants. Average MMSE scores were significantly different between groups with low CPS scores compared with those with high CPS scores $(p<0.05)$. CPS explained only $48.8 \%$ of the variability in MMSE. Agreement in defining cognitively impaired subjects was moderate (percentage observed agreement, 68\%; $\kappa=0.41$ ). With MMSE score less than 24 as a gold standard, diagnostic accuracy of CPS was moderate (area under curve $=0.73$ ), with low sensitivity, but excellent specificity. When lowering the MMSE cutoff to less than 18 and focusing on patients with severe cognitive impairment, CPS agreement coefficients and sensitivity increased but specificity decreased. Using education-adjusted MMSE cutoffs did not substantially affect the results. Conclusion: CPS can be used for coarse triage between intact and severe cognitive impairment. Although promising results have been obtained in residential and community settings, our results suggest that CPS fails to differentiate across different levels of cognitive impairment in hospitalized geriatric patients. (Am J Geriatr Psychiatry 2013; 21:636-645)
\end{abstract}

Key Words: Aged, geriatric assessment, inpatient, interRAI Acute Care, Minimum Data Set, validation studies

Received May 27, 2011; revised October 14, 2011; accepted November 30, 2011. From the Department of Public Health, Center for Health Services and Nursing Research, KU Leuven, Leuven, Belgium (NIHW, TH, PM, KM); Geriatric Medicine, University Hospitals Leuven \& Department of Clinical and Experimental Medicine, KU Leuven, Belgium (JF, JT, SB, KM); Interuniversity Institute for Biostatistics and Statistical Bioinformatics, KU Leuven, Leuven and Universiteit Hasselt, Hasselt, Belgium (GV). Send correspondence and reprint requests to Koen Milisen, Ph.D., R.N., or Nathalie I.H. Wellens, Ph.D., S.L.P., M.Sc., Center for Health Services and Nursing Research, Kapucijnenvoer 35-PB 7001/4, B-3000 Leuven, Belgium. e-mail: nathalie.wellens@med.kuleuven.be or koen.milisen@med.kuleuven.be

(C) 2013 American Association for Geriatric Psychiatry

http:/ /dx.doi.org/10.1016/j.jagp.2012.12.017 
C ognitive impairment is common in elderly inpatients. Acute cognitive decline is often a first indicator of an underlying somatic imbalance (e.g., dehydratation, urinary incontinence). ${ }^{1}$ Studies have shown cognitive impairment to be associated with increased mortality and morbidity, higher rehospitalization rates, and higher rates of cognitive and functional decline during ${ }^{2,3}$ and after hospitalization. $^{3-6}$ Diminished or altered cognitive functioning is often perceived as an inevitable consequence of the ageing process, and thus underrecognized. ${ }^{1,7}$ This may affect the effectiveness of treatment and interventions, and increase the length of hospitalization. ${ }^{1}$ Systematic cognitive screening of all older inpatients with subsequent detailed in-depth assessment and documentation of the cognitively impaired person may reduce the risk of adverse outcomes and delay institutionalization. ${ }^{8-10}$

Screening for cognitive dysfunction in the elderly population can be carried out by various instruments, including the Cognitive Performance Scale (CPS), a scale that is typically incorporated in a global geriatric assessment and evaluates cognitive functioning as a subdomain of overall functioning. It is based on a subset of five items of the interRAI/Minimum Data Set instruments and was originally developed to evaluate cognitive functioning in a residential population. ${ }^{11}$ In residential care, studies have shown good agreement between the Mini-Mental State Examination (MMSE) and CPS. ${ }^{11-16}$ In recent years, the interRAI portfolio has expanded to various care settings such as palliative care, acute care, post-acute care, and so forth. A key advantage of the interRAI portfolio is that it uses a uniform language across health professions and across care settings. ${ }^{17}$ On the basis of standardized items, (cognitive) functioning of the patient can be followed over time. This implies psychometric challenges, because effective communication in transitional care demands solid psychometric fundaments to rely on. Although promising results have been obtained in psychometric testing in residential care, performance of the CPS in the acute setting may be only moderate. ${ }^{18}$ In this context, psychometric research in acute care is an important research topic for several reasons. First, in various nations, the use of the interRAI portfolio, including the interRAI Acute Care (interRAI AC) instrument, has continued to grow. Second, to date, all studies that have tested the agreement between MMSE and
CPS have been based on the initial CPS algorithm. However, with the restructuring in 2005 of the interRAI Suite, CPS was modified into the $\mathrm{CPS}_{2}$ version. To the best of our knowledge, this is the first study to evaluate the performance of the $\mathrm{CPS}_{2}$ scale. Our aim was to explore agreement and interchangeability of the recent CPS as a part of the interRAI AC instrument with MMSE in patients admitted to acute geriatric wards.

\section{METHODS}

\section{Design and Sample}

An observational study was conducted using a convenience sample of patients admitted to two geriatric wards of the Leuven University Hospitals, Leuven, Belgium. Patients aged 75 years or older who were present on the predetermined assessment days between November 2009 and April 2010 were recruited consecutively between 24 and 48 hours after admission. Patients not speaking Dutch, not verbally testable, or transferred from another ward were excluded.

\section{Assessment of Cognitive Functioning}

Cognitive functioning at hospital admission was assessed by two trained assessors not directly involved in the care of the patient. One assessor completed the MMSE. ${ }^{19}$ The other assessor completed the comprehensive interRAI AC/Minimum Data Set. $^{20}$ The assessors were blinded to each others' results and were not allowed to exchange information. The order of the instruments (e.g., MMSE before interRAI AC and vice versa) was chosen at random.

MMSE scores range from 0 to 30, with higher scores reflecting better cognition. In clinical practice, traditionally patients are classified as cognitively impaired if scoring 23 or less, and classified as severe cognitively impaired if scoring 17 or less. ${ }^{21}$ To consider the potential effect from educational differences, ${ }^{21}$ additional analyses were performed on the basis of the $\leq 21$-point cutoff for the low-educated group (those who left school before the age of 16) and the $\leq 24$-point cutoff for the high-educated group (education after the age of 15 years) as proposed by Anderson et al. ${ }^{22}$ interRAI AC/Minimum Data Set was completed according to standard interRAI 
conventions, based on semistructured clinical interview, clinical observation, and chart review. The entire interRAI AC/Minimum Data Set (version 2009) was administered, consisting of 98 items organized in 11 domains (patient's history, cognition, communication, mood and behavior, functional status, continence, nutrition, health condition, diagnoses and medication, skin condition, discharge potential). The CPS is based on the patient's awakening status (comatose or not), and his or her performance in the following domains: ability in making decisions regarding tasks of daily life, shortterm memory, procedural memory, ability to make him or herself understood, and selfperformance in eating. The item scores are not summed, instead a computer-based algorithm (the $\mathrm{CPS}_{2}$ algorithm released by interRAI in 2006) is calculated. The purpose of the CPS is twofold. On the one hand, it aims to identify patients with cognitive impairment. In the current analysis, the usual cutoff of 2 points or more was used to define the presence of cognitive impairment. ${ }^{12}$ On the other hand, it aims to map fluctuations and severity of cognitive (dys) functioning by calculating scores ranging from 0 to 6 , with higher scores indicating higher cognitive impairment. ${ }^{11}$

In a 6-hour training program, the standardized scoring criteria of the interRAI AC manual ${ }^{23}$ and the use of the MMSE were explained to four assessors, and fictive cases were discussed. In addition, five reallife practice cases were independently scored on the basis of one interview and subsequently discussed to ensure that adequate agreement was attained. These were excluded from the study sample.

The study protocol and the written informed (proxy) consent procedure was approved by the Medical Ethics Committee of the Leuven University Hospitals, Belgium.

\section{Demographic and Clinical Variables}

Patient characteristics (age, gender, residential status, living arrangement) and clinical data (main reason for admission, medication, etc.) were collected using the interRAI AC.

\section{Statistical Analysis}

Descriptive statistics were expressed in percentages for nominal variables; means and standard deviations (or medians and quartiles in case of skewed distributions) were calculated for continuous variables. The association between MMSE and the CPS, the level of agreement between them, and the predicted value of CPS for MMSE were explored using correlations, analysis of variance (ANOVA), agreement coefficients, and diagnostic accuracy.

The Spearman correlation coefficient was calculated for the raw scores. One-way ANOVA analysis was used to compare the mean MMSE scores in the various CPS groups. Post-hoc pairwise comparisons are based on the Tukey HSD (honestly significant difference) post-hoc test.

The classification into "cognitive impaired" (e.g., MMSE $\leq 23$ and CPS $\geq 2$ versus "not cognitive impaired" (e.g.,MMSE $\geq 24$ and CPS $\leq 1$ ) was explored using observed agreement in tandem with $\kappa$ coefficients. The proportion of observed agreement $\left(P_{\mathrm{o}}\right)$ is the ratio of exact agreement between the two instruments in function of the total number of assessments. Cohen's $\kappa$ coefficient is a measure of agreement between two instruments, corrected for chance. Unweighted Cohen's $\kappa$ was used for the dichotomized variable "cognitive impairment." The strength of agreement for the $\kappa$ coefficient is according to Landis and Koch (1977) ${ }^{24}$ considered as poor for $\kappa$ values below 0.40 ; moderate from 0.41 to 0.60 ; substantial from 0.61 to 0.80 ; and above 0.81 almost perfect. For binary variables, paradoxes in agreement parameters (i.e., $\left.P_{\mathrm{O}}, \kappa\right)$ can be due to bias and prevalence effects. $^{25-27}$ The prevalence of scores affects the stability of $\kappa$. If the ratings of the sample of patients lack variability (i.e., are homogeneous), it is unlikely that $K$ will be close to the maximum score of 1 . This phenomenon is independent of the sample size. Therefore, the prevalence index is calculated ${ }^{28}$ : the absolute value of the difference between the number of cases rated as positive by both methods $A$ and $B$, and the number of cases rated as negative by both methods $\mathrm{A}$ and $\mathrm{B}$; divided by the total number of assessments.

Bias is the extent to which the methods disagree on the proportion of cases in a specific category. It may be identified by tendencies of methods to have systematically different classification patterns. Bias affects the interpretation of the magnitude of $K$. When the bias is large, $\kappa$ tends to be higher than when bias is low or absent. ${ }^{25,29}$ The bias index is calculated ${ }^{28}$ : the absolute value of the difference between the number of cases rated as positive by method A and negative by 
method $B$, and the number of cases rated as negative by method A and positive by method B; divided by the total number of assessments.

Diagnostic accuracy was calculated by sensitivity, specificity, positive and negative predictive value, likelihood ratio of a positive and a negative test, overall accuracy and area under the receiver operating characteristic curve (AUC) for the CPS with the original cutoff score $(\geq 2)$ and alternative cutoffs on 2-by-2 tables.

Additional analyses with corrections for education level were performed for 7 low-educated patients out of the total sample of 97 patients by lowering the cutoff to MMSE score of 21 or less, corrections were performed for 3 high-educated patients by increasing the cutoff to MMSE score of 24 or less (in stead of $\leq 23$ ).

All tests were two-sided and $\mathrm{p}<0.05$ was used as a level of significance. Analyses were performed with SPSS, version 17.

\section{RESULTS}

\section{Sample}

A total of 129 older patients admitted to hospital met the inclusion criteria. Eight patients were unwilling to participate. Nineteen were excluded because they were not verbally testable $(\mathrm{n}=16)$, because of insufficient comprehension of Dutch $(n=1)$, or infectious diseases requiring isolation $(n=2)$. Five patients discontinued the study because of very poor health condition (e.g., extreme pain, fatigue, dyspnoea). The mean age of the 97 included patients was 85.5 years, and the majority was female. Main reasons for admission were respiratory problems, functional decline, trauma/falls, and gastrointestinal problems (Table 1). The mean MMSE score was 19 (SD: 8) and cognitive impairment (e.g., MMSE $\leq 23$ ) was present in $61 \%$ of the participants. Participants with cognitive impairment as measured with the CPS did not differ from participants without cognitive impairment on demographic, social, and clinical characteristics, except for a significant difference in the mean MMSE score (Table 1).

\section{Convergent Validity of the CPS and MMSE}

Descriptive analyses and ANOVA. Overall, the higher the CPS level the lower the mean MMSE score
(Table 2). However, the mean MMSE scores for CPS levels 2, 3, and 4 were similar $(15 \pm 7,14 \pm 6,14 \pm 8$, respectively). These findings, based on descriptive analyses, were confirmed by ANOVA, showing an overall significant difference of mean MMSE score on levels of CPS score, $\left(F_{[6,90]}=14.3, \mathrm{p}<0.0001\right)$. Posthoc testing revealed that the mean of the group with CPS score 0 was not significantly different from the mean of the group with CPS score 1, but significant differences in means were observed with the groups with CPS scores 2-6. Furthermore, the mean of the group with CPS score 1 was not significantly different from the group with CPS scores 2 and 4, but it was significantly different from the group with CPS scores 3,5, and 6 . The mean of the group with CPS score 2 was not significantly different from the groups with CPS score $1-6$ but only significantly different from the group with CPS score 0 . The means of the groups of the CPS score 3, 5, and 6, were significantly different from the means of the group 0 and 1 . Some $49 \%$ of the variation in MMSE scores $\left(R^{2}\right)$ was explained by the CPS scores. The pooled within-groups standard deviation was 5.78, reflecting a large variation around the mean MMSE score within each CPS group. This is graphically presented in Figure 1.

Association and agreement between CPS and MMSE. Correlation between MMSE and CPS was moderate (Spearman $r=-0.60, \mathrm{p}<0.0001$ ). Agreement in defining cognitively impaired subjects was also moderate $\left(P_{\mathrm{o}}=68 \% ; \kappa=0.41, \mathrm{z}=5.33\right.$, $\mathrm{p}<0.0001,95 \%$ confidence interval [CI]: 0.26-0.55). The prevalence index was 0.06 . The bias index was 0.28 revealing that the disagreement was asymmetrical, supporting a tendency of the two cognitive tools to have systematically different classification patterns (McNemar $\mathrm{p}<0.0001) .{ }^{28}$ By using the education-adjusted cutoffs, similar results were obtained $\left(P_{\mathrm{o}}=71 \% ; \mathrm{K}=0.46, \mathrm{z}=5.71, \mathrm{p}<0.0001,95 \%\right.$ CI: $0.30-0.61)$, prevalence index $=0.08$, bias index $=0.24, \mathrm{p}<0.0001)$.

Diagnostic accuracy. With MMSE score less than 24 as a traditional gold standard for cognitive impairment, the overall diagnostic accuracy of the CPS at a cutoff 2 was moderate, with low sensitivity, but excellent specificity (Table 3). Lowering the CPS cutoff to 1 , increased the sensitivity but reduced the specificity. Increasing the CPS cutoff to 3 resulted in the opposite effect. When lowering the MMSE cutoff 
TABLE 1. Characteristics of Participants $(N=97)$

\begin{tabular}{|c|c|c|c|c|c|c|}
\hline \multirow[b]{2}{*}{ Characteristic } & \multirow[b]{2}{*}{ Total Sample $(\mathbf{N}=97)$} & \multicolumn{5}{|c|}{ Cognitive Impairment According to CPS } \\
\hline & & No $(n=65)$ & Yes $(n=32)$ & Test Value & $d f$ & $\mathbf{p}^{\mathbf{a}}$ \\
\hline Age, years $($ mean $\pm S D)$ & $85 \pm 5$ & $84 \pm 5$ & $86 \pm 5$ & $t=-1.59$ & 95 & $0.12^{\mathrm{b}}$ \\
\hline Gender, n (\%) & & & & $\chi^{2}=0.04$ & 1 & $0.84^{\mathrm{c}}$ \\
\hline Female & $65(67)$ & $44(68)$ & $21(66)$ & & & \\
\hline Male & $32(33)$ & $21(32)$ & $11(34)$ & & & \\
\hline Residential status, n (\%) & & & & $\chi^{2}=6.20$ & 2 & $0.05^{\mathrm{c}}$ \\
\hline Community & $77(79)$ & $56(86)$ & $21(65)$ & & & \\
\hline Nursing home & $15(15)$ & $6(9)$ & $9(28)$ & & & \\
\hline Other & $5(5)$ & $4(6)$ & $2(6)$ & & & \\
\hline Living arrangement, $\mathrm{n}(\%)$ & & & & $\chi^{2}=2.57$ & 3 & $0.46^{\mathrm{c}}$ \\
\hline Alone & $33(34)$ & $25(39)$ & $8(25)$ & & & \\
\hline With spouse or partner & $26(27)$ & $17(26)$ & $9(28)$ & & & \\
\hline With relatives & $13(13)$ & $9(14)$ & $4(13)$ & & & \\
\hline With non-relatives & $25(26)$ & $14(21)$ & $11(33)$ & & & \\
\hline $\begin{array}{l}\text { Total number of medications at admission, } \\
\text { median (Q1; Q3) [Range] }\end{array}$ & $9(6 ; 11)[1-21]$ & $8(7 ; 11)[1-21]$ & $9(4 ; 11)[2-17]$ & $U=966.50$ & & $0.57^{\mathrm{d}}$ \\
\hline Taking $\geq 5$ medications premorbid, $\mathrm{n}(\%)$ & $85(84)$ & $58(89)$ & $24(63)$ & $\chi^{2}=3.32$ & 1 & $0.07^{\mathrm{c}}$ \\
\hline Main reason for admission, $\mathrm{n}(\%)$ & & & & $\chi^{2}=4.98$ & 5 & $0.42^{\mathrm{c}}$ \\
\hline Trauma & $13(13)$ & $9(14)$ & $4(13)$ & & & \\
\hline Gastrointestinal & $10(10)$ & $5(8)$ & $5(16)$ & & & \\
\hline Cardiovascular & $1(1)$ & $0(0)$ & $1(3)$ & & & \\
\hline Respiratory & $25(26)$ & $19(29)$ & $6(20)$ & & & \\
\hline General decline & $20(21)$ & $12(18)$ & $8(25)$ & & & \\
\hline Other & $28(29)$ & $20(31)$ & $8(25)$ & & & \\
\hline Comorbidities, median (Q1; Q3) [Range] & $4(3 ; 5)[1-10]$ & $4(3 ; 5)[1-10]$ & $3(2 ; 5)[1-9]$ & $U=965.50$ & & $0.56^{\mathrm{d}}$ \\
\hline $\begin{array}{l}\text { Length of stay, median number of days } \\
\text { (Q1; Q3) [Range] }\end{array}$ & $14(9 ; 22)[3-52]$ & $12(9 ; 22)[3-52]$ & $16(11 ; 23)[4-48]$ & $U=859.50$ & & $0.17^{\mathrm{d}}$ \\
\hline \multicolumn{7}{|l|}{ Cognitive impairment at admission } \\
\hline Mean \pm SD MMSE score & $19 \pm 8$ & $23 \pm 5$ & $12 \pm 7$ & $t=8.14$ & 95 & $<0.0001^{b}$ \\
\hline Median (Q1; Q3) MMSE score & $21(13 ; 15)$ & $24(19 ; 27)$ & $12(6 ; 19)$ & $U=269.00$ & & $<0.0001^{\mathrm{d}}$ \\
\hline \multicolumn{7}{|c|}{$\begin{array}{l}\text { Notes: The completion rate was } 100 \% \text { for the MMSE and CPS items. Within the total data set of all interRAI AC items, } 10 \text { items showed } \\
\text { a missing record but only for } 1 \% \text { of the assessments. CPS cutoff } 2 \text {; MMSE cutoff } 24 \text {. Q1: first quartile; Q3: third quartile. } \\
\text { ap value comparing the group without cognitive impairment according to the CPS }(\leq 1) \text { and the group cognitive impaired according to the } \\
\text { CPS }(\geq 2) \text {. } \\
\text { b Independent samples } t \text {-tests. } \\
{ }^{c} \chi^{2} \text { tests. } \\
{ }^{\mathrm{d}} \text { Mann-Whitney } U \text { test. }\end{array}$} \\
\hline
\end{tabular}

(MMSE <18) and considering patients with severe cognitive impairment, agreement coefficients for the usual CPS cutoff $(\geq 2)\left(P_{\mathrm{o}}=80 \% ; \kappa=0.54, \mathrm{z}=6.01\right.$, $\mathrm{p}<0.0001,95 \%$ CI: .36-0.72), AUC (0.77) and sensitivity (0.68) increased with a specificity of 0.86 . By using the education-adjusted cutoffs, similar results were obtained (Table 3).

\section{DISCUSSION}

The aim of this study was to determine whether cognitive assessment by MMSE and CPS are in sufficient agreement to be used interchangeably in acute care. In medical inpatients, we were unable to replicate the good agreement previously shown between both methods in a nursing home population. Our results are in line with another study in an acute care setting, ${ }^{18}$ despite significant differences in the prevalence of cognitive impairment $(61 \%$ in our study versus $32 \%$ ) and despite the use of a different CPS algorithm (the recent $\mathrm{CPS}_{2}$ versus the initial CPS).

CPS scores were moderately correlated with MMSE scores and accounted for less than $50 \%$ of the variation in MMSE scores. This means that only a minor proportion of the variability in MMSE scores can be explained by CPS scores. Although significant differences in average MMSE between various CPS groups were documented in ANOVA analyses, we could not establish significant differences between each level of CPS score. Along the seven levels of CPS 
TABLE 2. Average MMSE Score, Percentage of Subjects, and Significant Differences of the Means for each Level on the Cognitive Performance Scale $(\mathbf{N}=97)$

\begin{tabular}{|c|c|c|c|c|c|c|c|c|c|}
\hline \multicolumn{2}{|c|}{ Cognitive Status According to CPS } & \multicolumn{4}{|c|}{ Average MMSE Score } & \multicolumn{2}{|c|}{ MMSE $\leq 23$} & \multicolumn{2}{|c|}{ MMSE $\leq \mathbf{1 7}$} \\
\hline Score & Label & $\mathbf{n}$ & Mean & SD & Tukey HSD ${ }^{\mathbf{a}}$ & $\mathbf{n}$ & $\%^{\mathbf{b}}$ & $\mathbf{n}$ & $\% \mathrm{c}$ \\
\hline 0 & Intact & 42 & 23 & 5 & A & 16 & 38 & 5 & 12 \\
\hline 1 & Borderline intact & 23 & 21 & 6 & $\mathrm{AB}$ & 13 & 57 & 6 & 26 \\
\hline 2 & Mild impairment & 7 & 15 & 7 & $\mathrm{BC}$ & 6 & 86 & 4 & 57 \\
\hline 3 & Moderate impairment & 12 & 14 & 6 & $\mathrm{C}$ & 11 & 92 & 8 & 67 \\
\hline 4 & Moderate severe impairment & 5 & 14 & 8 & $\mathrm{BC}$ & 5 & 100 & 3 & 60 \\
\hline 5 & Severe impairment & 6 & 6 & 6 & $\mathrm{C}$ & 6 & 100 & 6 & 100 \\
\hline 6 & Very severe impairment & 2 & 6 & 8 & $\mathrm{C}$ & 2 & 100 & 2 & 100 \\
\hline $0-1$ & No cognitive impairment & 65 & 23 & 5 & & 29 & 45 & 11 & 38 \\
\hline $2-6$ & Cognitive impairment & 32 & 12 & 7 & & 30 & 94 & 23 & 72 \\
\hline Total & & 97 & 19 & 8 & & 59 & 61 & 34 & 35 \\
\hline
\end{tabular}

${ }^{a} A, B, C$ refer to significant differences of the means between the groups for each level of CPS ( $<<0.05$ ). Groups with equal letters have average MMSE values, which are not significantly different from one another.

${ }^{b}$ Proportion of the number of persons with MMSE $\leq 23$ and the total number of persons for each level of CPS score.

'Proportion of the number of persons with MMSE $\leq 17$ and the total number of persons for each level of CPS score.

FIGURE 1. Mean MMSE score and confidence interval of the mean for each level of the CPS score. Statistical significance of the differences between each group per level of CPS was tested by the Tukey HSD post-hoc test. A, B, C refer to significant differences between the groups for each level of CPS $(p<0.05)$. Groups with equal letters have average MMSE values, which are not significantly different from one another. $95 \%$ ci: $95 \%$ confidence interval based on the pooled SD (5.78) and $d f(6,90)$.

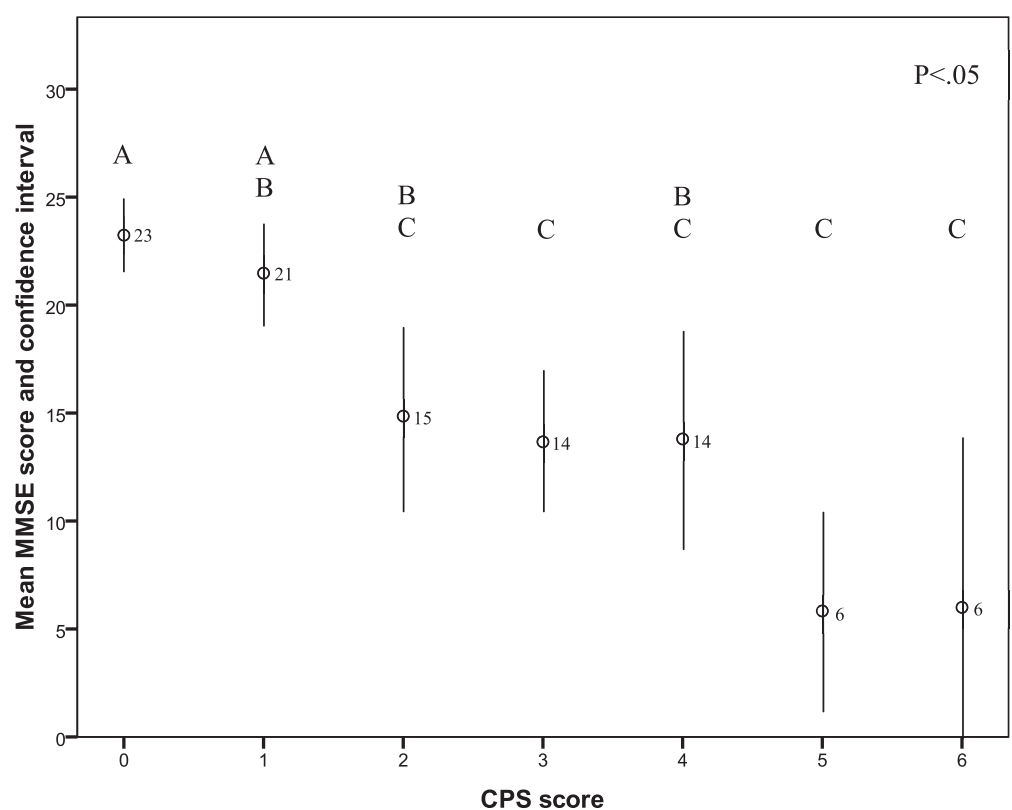

\begin{tabular}{|l|c|c|c|c|c|c|c|}
\hline Mean MMSE & 23 & 21 & 15 & 14 & 14 & 6 & 6 \\
\hline$[\mathrm{LB}-\mathrm{UB}]$ & {$[21.47-$} & {$[19.09-$} & {$[10.52-$} & {$[10.35-$} & {$[8.66-$} & {$[1.14-$} & {$[-2.12-$} \\
& $25.01]$ & $23.87]$ & $19.20]$ & $16.99]$ & $18.94]$ & $10.52]$ & $14.12]$ \\
\hline
\end{tabular}




\begin{tabular}{|c|c|c|c|c|c|c|c|c|c|}
\hline Instrument & Cutoff & Sens (\%) & Spec (\%) & PPV (\%) & NPV (\%) & $\operatorname{Acc}(\%)$ & $\mathbf{L H}+$ & LH - & AUC \\
\hline \multicolumn{10}{|l|}{ MMSE $<24$} \\
\hline \multirow[t]{5}{*}{ CPS } & 1 & 0.73 & 0.68 & 0.78 & 0.62 & 0.71 & 2.3 & 0.40 & 0.71 \\
\hline & 2 & 0.51 & 0.95 & 0.94 & 0.55 & 0.68 & 9.67 & 0.52 & 0.73 \\
\hline & 3 & 0.41 & 0.97 & 0.96 & 0.51 & 0.63 & 15.5 & 0.61 & 0.69 \\
\hline & 4 & 0.22 & 1.00 & 1.00 & 0.45 & 0.53 & - & 0.78 & 0.61 \\
\hline & 5 & 0.14 & 1.00 & 1.00 & 0.43 & 0.47 & - & 0.86 & 0.57 \\
\hline \multicolumn{10}{|l|}{ MMSE $<18$} \\
\hline \multirow[t]{5}{*}{ CPS } & 1 & 0.85 & 0.59 & 0.53 & 0.88 & 0.68 & 2.07 & 0.25 & 0.72 \\
\hline & 2 & 0.68 & 0.86 & 0.72 & 0.83 & 0.80 & 4.74 & 0.38 & 0.77 \\
\hline & 3 & 0.56 & 0.91 & 0.76 & 0.79 & 0.78 & 5.87 & 0.49 & 0.73 \\
\hline & 4 & 0.32 & 0.97 & 0.85 & 0.73 & 0.74 & 10.2 & 0.70 & 0.65 \\
\hline & 5 & 0.24 & 1.00 & 1.00 & 0.71 & 0.73 & - & 0.77 & 0.62 \\
\hline MMSE adj. ${ }^{a}$ & 2 & 0.54 & 0.95 & 0.94 & 0.60 & 0.71 & 10.40 & 0.48 & 0.75 \\
\hline
\end{tabular}

\footnotetext{
Notes: Sens: sensitivity; Spec: specificity; PPV: positive predictive value; NPV: negative predictive value; Acc: accuracy; LH+: likelihood ratio of positive test; $\mathrm{LH}-$ : likelihood ratio of negative test.

${ }^{a}$ MMSE Adj.: MMSE cutoff scores adjusted for education $\leq 21$ for those with low educational level (i.e., those who left school before the age of 16) and $\leq 24$ for those with high educational level (i.e. education after the age of 15); educational level missing for 6 subjects, analyses based on $\mathrm{n}=91$.
}

scores, only three subgroups were identified, with overlapping CPS score. The implication of these findings is that CPS only discriminates between patients with intact cognitive functioning and severe cognitive impairment, but fails to differentiate across the full spectrum of cognitive impairment. In addition, the within-group standard deviation was large, reflecting substantial variation in MMSE scores within each CPS group. These considerable discrepancies between MMSE and CPS scores are clinically unacceptable.

Agreement between MMSE and CPS was also examined by $\kappa$ coefficients, again showing moderate agreement between the two methods, for both the traditional and the education-adjusted cutoff. Although this result was not biased by homogeneity in the sample, it is biased by a systematically different classification pattern between the two cognitive tools. ${ }^{28}$ As a result, the magnitude of $\kappa$ is higher, meaning that it must be interpreted with caution. ${ }^{29}$

This trend of asymmetric disagreement between the MMSE and CPS was confirmed by a moderate sensitivity despite excellent specificity, resulting in a moderate AUC when the typical CPS cutoff advised was used. Lowering the cutoff value to 1 would identify more cognitively impaired inpatients and also increase the number of false positives. On the basis of these findings, the ideal cutoff value of the CPS remains uncertain. Shifting the cutoff of the gold standard to severe cognitive impairment (MMSE $<18$ ), the agreement improved on various parameters except for a slight decrease in specificity, suggesting that CPS and MMSE may be more in agreement when detecting the severe cognitive impaired inpatients. Correction for the level of education did not substantially affect these results. Whatever the types of analyses, it would seem that CPS can be used for coarse triage, but not for differentiation between different levels of cognitive functioning, nor for detailed mapping or follow-up through different levels of cognitive impairment. In other words, these results suggest that if the interRAI portfolio is used in transitional care, CPS scores cannot be validly transferred from one setting to another to track the patients' functioning longitudinally.

In the study of Büla and Wietlisbach, ${ }^{18}$ the only other study in an acute setting, findings were generally similar. Sensitivity and specificity values were in line with those reported in this study. However, different results were found for the mean values of the MMSE for each level of CPS, with an increasing trend in MMSE scores for decreasing CPS scores. The use of a different algorithm to calculate CPS cannot explain this discrepancy, because our analyses yielded similar results when $\mathrm{CPS}_{2}$ was replaced by the $\mathrm{CPS}_{1}$ (data not shown). It is therefore likely that differences in sample size, data collection, and/or studied population may have been important. In the study by Büla and Wietlisbach, ${ }^{18}$ data were collected by a researcher interviewing the responsible nurse whereas our data 
collection was based on comprehensive assessment and chart review by researchers.

Nevertheless, and despite some inconsistencies between studies, it would seem that MMSE and CPS show less agreement in an acute setting than in a residential setting ${ }^{12,15,16,30-35}$ or community ${ }^{36}$ care, with reported correlations between -0.40 and -0.88 , sensitivity levels between 0.81 and 0.94 , specificity between 0.80 and 1.00 , and $\kappa$ values between 0.85 and 0.88 , respectively. ${ }^{12,15,16,30-36}$ If and to what extent differences in performance of CPS between residential or community settings and acute care is due to differences in population characteristics, underlying acute illnesses, and/or differences in CPS algorithms, remains to be clarified.

Our study has strengths and limitations. Strength of this study was the range of parameters and analyses used to evaluate the association, agreement, and diagnostic value, supporting the validity of our results. The use of correlation coefficients to assess the validity of an instrument is disputed because it can be misleading. Correlations are dependent on the range of measurements, on the way the sample was chosen, and typically ignore any systematic difference between the instruments under study. ${ }^{37-39}$ Nevertheless, most previous validity studies of the interRAI/MDS instruments have relied on correlation coefficients only. If we had only used a similar approach, we might have concluded that MMSE and CPS show good agreement (Spearman $r=-0.60$, $\mathrm{p}$ $<0.0001)$.More profound analyses showed a different picture, as discussed. Finally, the analyses based on dichotomous classification of the patients were both performed by the traditional MMSE cutoff and education-adjusted cutoffs. The former allowed comparison with previous research on the CPS, the latter attempts to decrease the potential for misclassification. ${ }^{21,22}$ A limitation, on the contrary, was the lack of a gold standard for the evaluation of cognitive functioning, which may have led to an artifact. Because cognitive functioning cannot be directly measured, both instruments attempt to indirectly quantify a latent construct. We assessed the agreement between two methods but could not address the underlying question if CPS adequately measures (different levels of) cognitive functioning. Although MMSE is widely used as a reference, it is by no means a perfect standard of cognitive functioning. Also, because using MMSE comes with practical limitations, some patients with severe illness or severe confusion are typically excluded, which may result in sampling bias. Finally, prevalence of cognitive impairment in our study was larger (62\%) than expected for this type of population, ${ }^{5,40,41}$ probably because patients were recruited on geriatric wards, instead of general medical wards. We cannot exclude the possibility that this high prevalence may have affected the results.

The development of the interRAI instruments and embedded scales are an ongoing process and we suggest that more research is needed to adapt the CPS algorithm to the acute setting and to optimize CPS data transfer across settings. Ultimately, an adjusted CPS scale should not only be applicable in the acute setting, but also be exchangeable across clinical settings. To this end, CPS patient data in various settings will need to be obtained.

The demand to gather data of geriatric patients for research, ${ }^{42}$ and for regulatory and monitoring purposes is growing rapidly. Widespread implementation of the interRAI portfolio would imply possibilities of large-scale benchmarking making reliability, validity, and feasibility studies become essential. In addition to data obtained with the inter- RAI AC, ${ }_{1}^{18,20,43,44}$ a spectrum of clinimetric testing will have to be carried out to get a wide body of evidence. ${ }^{45,46}$

\section{CONCLUSIONS}

In an acute setting, the CPS derived from the interRAI AC/Minimum Data Set is able to discriminate between cognitively intact patients and those with severe cognitive impairment. However, unlike promising results in residential and community settings, the CPS only performs moderately in differentiating between different levels of cognitive impairment in older inpatients.

The authors thank the staff and the participating patients of the Geriatric wards of the University Hospitals Leuven, whose cooperation was essential and much appreciated. Dr. Boonen is senior clinical investigator of the Fund for Scientific Research (FWO-Vlaanderen) and holder of the Leuven University Chair in Gerontology and Geriatrics.

The authors declare that they have not received funding for the research of this article.

The authors have no conflicts of interest. 


\section{References}

1. Milisen $\mathrm{K}$, Braes T, Fick DM, et al: Cognitive assessment and differentiating the 3 Ds (dementia, depression, delirium). Nurs Clin North Am 2006; 41:1-22

2. Mecocci P, von Strauss E, Cherubini A, et al: Cognitive impairment is the major risk factor for development of geriatric syndromes during hospitalization: results from the GIFA study. Dement Geriatr Cogn Disord 2005; 20:262-269

3. Sands LP, Yaffe K, Covinsky K, et al: Cognitive screening predicts magnitude of functional recovery from admission to 3 months after discharge in hospitalized elders. J Gerontol A Biol Sci Med Sci 2003; 58:37-45

4. Arfken CL, Lichtenberg PA, Tancer ME: Cognitive impairment and depression predict mortality in medically ill older adults. J Gerontol A Biol Sci Med Sci 1999; 54:M152-M156

5. Joray S, Wietlisbach V, Bula CJ: Cognitive impairment in elderly medical inpatients: detection and associated six-month outcomes. Am J Geriatr Psychiatry 2004; 12:639-647

6. Pitkala KH, Laurila JV, Strandberg TE, et al: Prognostic significance of delirium in frail older people. Dement Geriatr Cogn Disord 2005; 19:158-163

7. Treloar AJ, Macdonald AJ: Recognition of cognitive impairment by day and night nursing staff among acute geriatric patients. J R Soc Med 1995; 88:196-198

8. Boustani M, Baker MS, Campbell N, et al: Impact and recognition of cognitive impairment among hospitalized elders. J Hosp Med $2010 ; 5: 69-75$

9. Flaherty JH, Rudolph J, Shay K, et al: Delirium is a serious and under-recognized problem: why assessment of mental status should be the sixth vital sign. J AmMed Dir Assoc 2007; 8:273-275

10. McCusker J, Cole M, Dendukuri N, et al: Delirium in older medical inpatients and subsequent cognitive and functional status: a prospective study. CMAJ 2001; 165:575-583

11. Morris JN, Fries BE, Mehr DR, et al: MDS cognitive performance scale. J Gerontol 1994; 49:M174-M182

12. Hartmaier SL, Sloane PD, Guess HA, et al: Validation of the minimum data set cognitive performance scale: agreement with the mini-mental state examination. J Gerontol A Biol Sci Med Sci 1995; 50:M128-M133

13. Hawes C, Morris JN, Phillips CD, et al: Reliability estimates for the Minimum Data Set for nursing home resident assessment and care screening (MDS). Gerontologist 1995; 35:172-178

14. Lawton MP, Casten R, Parmelee PA, et al: Psychometric characteristics of the minimum data set II: validity. $\mathrm{J}$ Am Geriatr Soc 1998; 46:736-744

15. Paquay L, De Lepeleire J, Schoenmakers B, et al: Comparison of the diagnostic accuracy of the Cognitive Performance Scale (Minimum Data Set) and the Mini-Mental State Exam for the detection of cognitive impairment in nursing home residents. Int J Geriatr Psychiatry 2007; 22:286-293

16. Snowden M, McCormick W, Russo J, et al: Validity and responsiveness of the Minimum Data Set. J Am Geriatr Soc 1999; 47: 1000-1004

17. Wellens NIH, Deschodt M, Flamaing J, et al: First-generation versus third-generation comprehensive geriatric assessment instruments in the acute hospital setting: a comparison of the Minimum Geriatric Screening Tools (MGST) and the interRAI Acute Care (interRAI AC). J Nutr Health Aging 2011; 15:638-644

18. Büla CJ, Wietlisbach V: Use of the Cognitive Performance Scale (CPS) to detect cognitive impairment in the acute care setting: concurrent and predictive validity. Brain Res Bull 2009; 80: $173-178$
19. Folstein MF, Folstein SE, McHugh PR: "Mini-mental state". A practical method for grading the cognitive state of patients for the clinician. J Psychiatr Res 1975; 12:189-198

20. Gray LC, Bernabei R, Berg K, et al: Standardizing assessment of elderly people in acute care: the interRAI Acute Care instrument. J Am Geriatr Soc 2008; 56:536-541

21. Tombaugh TN, McIntyre NJ: The mini-mental state examination: a comprehensive review. J Am Geriatr Soc 1992; 40:922-935

22. Anderson TM, Sachdev PS, Brodaty H, et al: Effects of sociodemographic and health variables on Mini-Mental State Exam scores in older Australians. Am J Geriatr Psychiatry 2007; 15:467-476

23. Gray L, Berg K, Bernabei R, et al: Training manual and resource guide InterRAI Acute Care: Guide for use of the interRAI AC assessment form (version 09). interRAI 2006

24. Landis JR, Koch GG: The measurement of observer agreement for categorical data. Biometrics 1977; 33:159-174

25. Byrt T, Bishop J, Carlin JB: Bias, prevalence and kappa. J Clin Epidemiol 1993; 46:423-429

26. Hoehler FK: Bias and prevalence effects on kappa viewed in terms of sensitivity and specificity. J Clin Epidemiol 2000; 53: 499-503

27. Vach W: The dependence of Cohen's kappa on the prevalence does not matter. J Clin Epidemiol 2005; 58:655-661

28. Sim J, Wright CC: The kappa statistic in reliability studies: use, interpretation, and sample size requirements. Phys Ther 2005; 85:257-268

29. Feinstein AR, Cicchetti DV: High agreement but low kappa: I. The problems of two paradoxes. J Clin Epidemiol 1990; 43: 543-549

30. Achterberg W, Pot AM, van Campen C, et al: [Resident Assessment Instrument (RAI): a review of international research on the psychometric qualities and effects of implementation in nursing homes]. Tijdschr Gerontol Geriatr 1999; 30:264-270

31. Chodosh J, Edelen MO, Buchanan JL, et al: Nursing home assessment of cognitive impairment: development and testing of a brief instrument of mental status. J Am Geriatr Soc 2008; 56 : 2069-2075

32. Frederiksen K, Tariot P, De Jonghe E: Minimum Data Set Plus (MDS +) scores compared with scores from five rating scales. J Am Geriatr Soc 1996; 44:305-309

33. Gruber-Baldini AL, Zimmerman SI, Mortimore E, et al: The validity of the minimum data set in measuring the cognitive impairment of persons admitted to nursing homes. J Am Geriatr Soc 2000; 48 : 1601-1606

34. Hartmaier SL, Sloane PD, Guess HA, et al: The MDS Cognition Scale: a valid instrument for identifying and staging nursing-home residents with dementia using the minimum data set. J Am Geriatr Soc 1994; 42:1173-1179

35. Van der Steen JT, Volicer L, Gerritsen DL, et al: Defining severe dementia with the Minimum Data Set. Int J Geriatr Psychiatry 2006; 21:1099-1106

36. Landi F, Tua E, Onder G, et al: Minimum data set for home care: a valid instrument to assess frail older people living in the community. Med Care 2000; 38:1184-1190

37. Bland JM, Altman DG: Statistical methods for assessing agreement between two methods of clinical measurement. Lancet 1986; 1: 307-310

38. Bland JM, Altman DG: Measuring agreement in method comparison studies. Stat Methods Med Res 1999; 8:135-160

39. Bland JM, Altman DG: Applying the right statistics: analyses of measurement studies. Ultrasound Obstet Gynecol 2003; 22:85-93 
40. Bowler C, Boyle A, Branford M, et al: Detection of psychiatric disorders in elderly medical inpatients. Age Ageing 1994; 23:307-311

41. Hickey A, Clinch D, Groarke EP: Prevalence of cognitive impairment in the hospitalized elderly. Int J Geriatr Psychiatry 1997; 12:27-33

42. Van Craen $\mathrm{K}$, Braes $\mathrm{T}$, Wellens $\mathrm{N}$, et al: The effectiveness of inpatient geriatric evaluation and management units: a systematic review and meta-analysis. J Am Geriatr Soc 2010; 58:83-92

43. Wellens NIH, Deschodt M, Boonen S, et al: Validity of the interRAI Acute Care based on test content: a multi-center study. Aging Clin Exp Res 2011; 23:476-486
44. Wellens NIH, Van Lancker A, Flamaing J, et al: Interrater reliability of the interRAI Acute Care. Arch Gerontol Geriatr 2012; 55:165-172

45. Wellens NIH, Milisen K, Flamaing J, et al: Methods to assess the validity of the interRAI Acute Care: a framework to guide clinimetric testing. J Eval Clin Pract 2012; 18:296-306

46. Wellens NIH, Milisen $\mathrm{K}$, Flamaing $\mathrm{J}$, et al: Methods to assess the reliability of the interRAI Acute Care: a framework to guide clinimetric testing. Part II. J Eval Clin Pract 2012; 18: $822-827$ 